\title{
II JPTM
}

Journal of Pathology and Translational Medicine 2021; 55: 161-162

\section{What's new in gynecologic pathology 2021: vulva, cervix, and uterus}

\author{
Carlos Parra-Herran ${ }^{1}$ and Jennifer A. Bennett ${ }^{2}$
}

'Department of Pathology, Brigham and Women's Hospital and Harvard Medical School, Boston, MA, USA

2Department of Pathology, University of Chicago Medical Center, Chicago, IL, USA

Received: March 8, 2021 Accepted: March 9, 2021 Corresponding Author: Carlos Parra-Herran, MD Department of Pathology, Brigham and Women's Hospital and Harvard Medical School, Boston, Massachusetts, USA

E-mail: CPARRA-HERRAN@bwh.harvard.edu

\section{ORCID}

\section{Carlos Parra-Herran}

https://orcid.org/0000-0003-1420-7291

Jennifer Bennett

https://orcid.org/0000-0001-8155-7131

This article has been published jointly, with consent, in both Journal of Pathology and Translational Medicine and PathologyOutlines.com

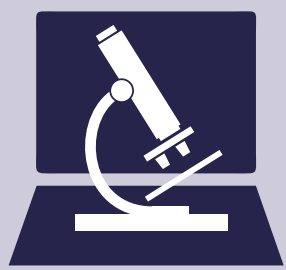

PathologyOutlines.com

The 5th edition of the World Health Organization of Tumours of Female Reproductive Organs was published in 2020. This is a compilation of the most important changes in the vulva, cervix and uterus.

\section{VULVA}

- Squamous intraepithelial lesions and squamous cell carcinomas are now classified as HPV-associated and HPV-independent.

- p16 overexpression is a reliable surrogate marker of HPV infection. A second marker with prognostic utility is $\mathrm{p} 53$. Thus, squamous neoplasms are now classified in three main groups: p16 overexpressed / p53 normal, p16 negative / p 53 abnormal and p16 negative / p53 normal.

- HPV-independent (p16 negative), p53 abnor- mal carcinomas have been associated with worse progression-free and overall survival compared to HPV-associated carcinomas as well as p16 negative / p 53 normal carcinomas.

- HPV-associated squamous intraepithelial lesions (formerly known as vulvar intraepithelial neoplasia of the usual / classic type) represent the majority (90\%) of precursors. They are referred to as low-grade (LSIL, equivalent to uVIN1) and high-grade (HSIL, equivalent to uVIN2 and uVIN3).

- HSIL is characterized by p16 overexpression and wild-type p 53 (with strong staining in mid-epithelial layers and negative or patchy basal / parabasal staining).

- The most common HPV-independent lesion is differentiated vulvar intraepithelial neoplasia (dVIN). Its presumed rapid progression to invasive carcinoma and the difficulties in its diagnosis likely explain why dVIN represents only $<10 \%$ of squamous intraepithelial lesions. - dVIN is characterized by negative or patchy p16 and mutant-type p53 expression (the latter could be full-thickness strong, basal strong, completely negative or cytoplasmic staining).

- Another, far less common form of HPV-independent lesion is the now called differentiated exophytic vulvar intraepithelial lesion (DE-VIL). This lesion demonstrates verruciform acanthosis, hypogranulosis and cytoplasmic pallor (Figure 1). Unlike HSIL and dVIN, DE-VIL lacks cytologic atypia.

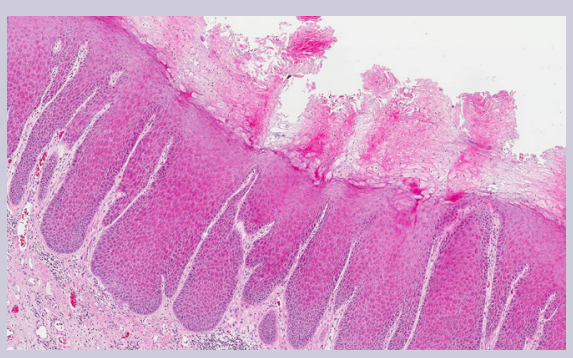

Fig. 1. Differentiated exophytic vulvar intraepithelial lesion (DE-VIL).
- DE-VIL has been associated with p16 negative / p 53 normal carcinomas, including verrucous and conventional squamous cell carcinoma.

- DE-VIL is characterized by negative or patchy p16 and wild-type p53 (heterogeneous staining).

\section{CERVIX}

- Carcinomas in both squamous and glandular categories are now classified as HPV-associated and HPV-independent.

- More than $90 \%$ of squamous cell carcinomas of the cervix are secondary to HPV infection. There is emerging evidence showing that the HPV-independent subgroup ( 7\%) has worse outcome.

- There are currently no morphologic clues to distinguish between HPV-associated and HPV-independent squamous cell carcinomas.

- Adenocarcinoma in-situ also now has two recognized categories: HPV-associated (frequently referred to as "usual") and HPVindependent. Currently, only gastric-type AIS and atypical lobular endocervical glandular hyperplasia belong to the latter category. They feature foamy clear to eosinophilic mucinous cytoplasm, distinct cell borders, nuclear atypia and intraglandular growth (tufting, micropapillary, cribriform) (Figure 2).

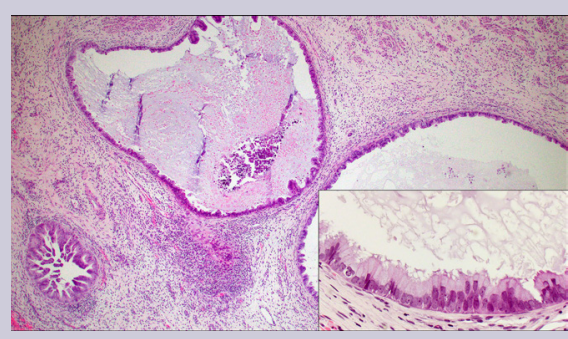

Fig. 2. Adenocarcinoma in situ, gastric type.

- HPV-independent adenocarcinomas, in particular gastric-type adenocarcinoma, have worse clinical behavior than HPV-associated adeno- 
carcinomas.

- HPV-associated endocervical adenocarcinomas represent $85-90 \%$ of all adenocarcinomas. Their histologic hallmark is the presence of conspicuous apical mitoses and apoptosis.

- They are characterized by p16 overexpression (strong and diffuse, nuclear and cytoplasmic staining), normal p53 staining and negative to weak ER/PR staining.

- The usual subtype is the most common, defined as having $<50 \%$ of cells with intracytoplasmic mucin, with the remaining having non-mucinous cytoplasm.

- The mucinous subtype, defined as having > $50 \%$ cells with intracytoplasmic mucin, can feature endocervical-type mucinous epithelium or intestinal-type epithelium.

- Invasive stratified mucin-producing carcinoma is a novel subtype, characterized by solid nests of multilayered mucinous epithelium resembling stratified mucin-producing intraepithelial lesion (SMILE). This subtype appears to have a worse outcome compared to other HPV-associated subtypes.

- Gastric-type adenocarcinoma represents the most common type of HPV-independent adenocarcinoma ( $10 \%$ of all adenocarcinomas). It includes the formerly called minimaldeviation adenocarcinoma / adenoma malignum.

- It features epithelium with foamy clear to eosinophilic mucinous cytoplasm and prominent cell borders.

- It is negative for hormone receptors, but often expresses HIK1083 (a marker of pyloric-type epithelium). p 53 can show mutant-type expression, and p16 can be strong and diffuse.

- Other recognized types of HPV-independent adenocarcinoma are clear cell and mesonephric. Endometrioid and serous carcinomas of the cervix are exceedingly rare, and their diagnosis requires first exclusion of an endometrial and tubo-ovarian primary.

\section{UTERUS-EPITHELIAL}

- An algorithm for applying The Cancer Genome Atlas (TCGA) classification system (POLEmutated, microsatellite instable, copy number low and copy number high/TP53-mutated) is provided. Surrogate markers for molecularly classifying endometrial carcinomas include targeted POLE sequencing, and MSH6, PMS2 and p53 immunohistochemistry.

- Molecular features in serous carcinomas have been updated to include ERBB2 (HER2) amplification in $30 \%$ of tumors; such patients have been shown to benefit from trastuzumab therapy.

- The category of mucinous carcinoma, defined by mucinous cells involving $>50 \%$ of the tumor, has been eliminated.

- Novel subtypes of endometrial carcinoma including mesonephric-like carcinoma and gastric (gastrointestinal)-type mucinous carcinoma have been added.
- Mesonephric-like carcinoma is characterized by a variety of architectural patterns, focal intraluminal eosinophilic secretions and nuclei resembling papillary thyroid carcinoma with mild to moderate atypia (Figure 3). They are often GATA-3, TTF-1, calretinin, and CD10 (luminal) positive, with ER negative or at most focally positive. Many harbor KRAS mutations and gain of chromosome 1q.

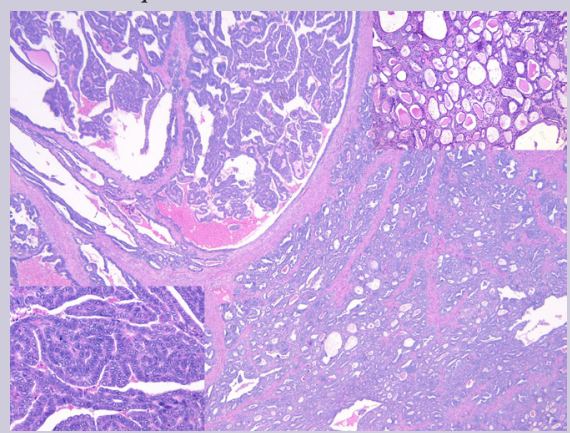

Fig. 3. Mesonephric-like endometrial carcinoma.

- Gastrointestinal-type mucinous carcinoma is composed of mucin-secreting glands (+/goblet cells) with low-grade atypia and may be mismatch repair protein-deficient.

- Carcinosarcoma is now considered a subtype of endometrial carcinoma rather than a mixed epithelial and mesenchymal tumor.

\section{UTERUS-MESENCHYMAL}

- Fumarate hydratase-deficient leiomyoma has been added as a subtype of leiomyoma and is characterized by staghorn vessels, alveolar-pattern edema, scattered bizarre nuclei, ovoid nuclei sometimes arranged in chains, eosinophilic cytoplasmic (rhabdoid) inclusions and prominent eosinophilic nucleoli surrounded by perinucleolar halos (Figure 4). It may harbor somatic or germline fumarate hydratase mutations, the latter being diagnostic of hereditary leiomyomatosis and renal cell carcinoma (HLRCC) syndrome.

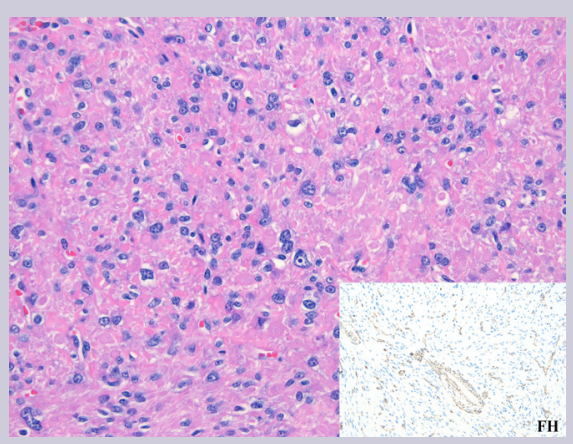

Fig. 4. Fumarate hydratase deficient leiomyoma.

- Specific diagnostic criteria for myxoid leiomyosarcoma are proposed: presence of any cytological atypia, tumor cell necrosis or $>1$ mitoses $/ 10$ HPF.
- IFITM1 has been included as a novel immunohistochemical stain that is often strongly and diffusely positive in endometrial stromal nodules and low-grade endometrial stromal sarcomas.

- Novel subtypes of high-grade endometrial stromal sarcoma include those with BCOR alterations (ZC3H7B-BCOR fusions or BCOR internal tandem duplication), which have a morphology overlapping with other myxoid mesenchymal neoplasms.

- ZC3H7B-BCOR sarcomas are positive for cyclin D1, CD10 and BCOR ( 50\%), with variable ER/PR.

- BCOR internal tandem duplication sarcomas are positive for cyclin D1 and BCOR, variably express $\mathrm{CD} 10$ and desmin, and are negative for ER, PR, SMA and caldesmon.

- Molecular alterations for uterine tumor resembling ovarian sex cord tumor (UTROSCT) include NCOA1-3, ESR1 or GREB1 fusions.

- Modified gynecologic-specific criteria for predicting PEComa behavior with elimination of the "benign" category are proposed.

- Inflammatory myofibroblastic tumor has been added as a distinct entity and a subset with malignant behavior is recognized. The most common ALK fusion partners include IGFBP5, THBS1 and TIMP3.

- NTRK-rearranged spindle cell neoplasm is a novel low-grade sarcoma most common in the cervix. It is positive for S100, CD34 and TRK, negative for ER, PR, CD10, SMA and desmin, and harbors NTRK fusions.

This newsletter is a featured collaboration with PathologyOutlines.com

\section{Conflicts of Interest}

The authors declare that they have no potential conflicts of interest.

\section{Meet the Authors}

Dr. Parra-Herran has been part of the Pathology Outlines editorial board since 2016. He is originally from Colombia and started his academic pathology career in Canada. He is currently affiliated with Brigham and Women's Hospital and Harvard Medical School as a pathologist and associate professor of pathology.

Dr. Bennett has been part of the Pathology Outlines editorial board since 2019 and was appointed deputy editor-in-chief of gynecologic pathology in 2020. She is currently an assistant professor at the University of Chicago Medical Center. Her research primarily focuses on the integration of morphology and molecular features, particularly in uterine mesenchymal neoplasms. 